Supplementary Information

\title{
Design of Molecular Scaffolds Based on Unusual Geometries for Magnetic Modulation of Spin-diverse Complexes with Selective Redox Response
}

\author{
Maurício Lanznaster, ${ }^{\dagger, \|}$ Mary Jane Heeg, ${ }^{\dagger}$ Gordon T. Yee, ${ }^{\ddagger}$ \\ Bruce R. McGarvey, ${ }^{\S, *}$ Claudio N. Verani ${ }^{\dagger}, *$ \\ ${ }^{\dagger}$ Department of Chemistry, Wayne State University - 5101 Cass Ave. Detroit, MI 48202 \\ * Department of Chemistry Virginia Polytechnic Institute and State University - 107 \\ Davidson Hall Blacksburg, VA 24061 \\ ${ }^{\S}$ Department of Chemistry and Biochemistry, University of Windsor - 401 Sunset Ave. \\ Windsor, ON N9B 1P4, Canada
}


Figure S1. Two different views for the overlap of the least-squares fits for $\mathbf{2}$ and $\mathbf{3}$ Dihedral angles between the outer rings of the phenanthroline ligands are $9.5(2)^{\circ}$ for 2 and 1.5(5) ${ }^{\circ}$ for 3.

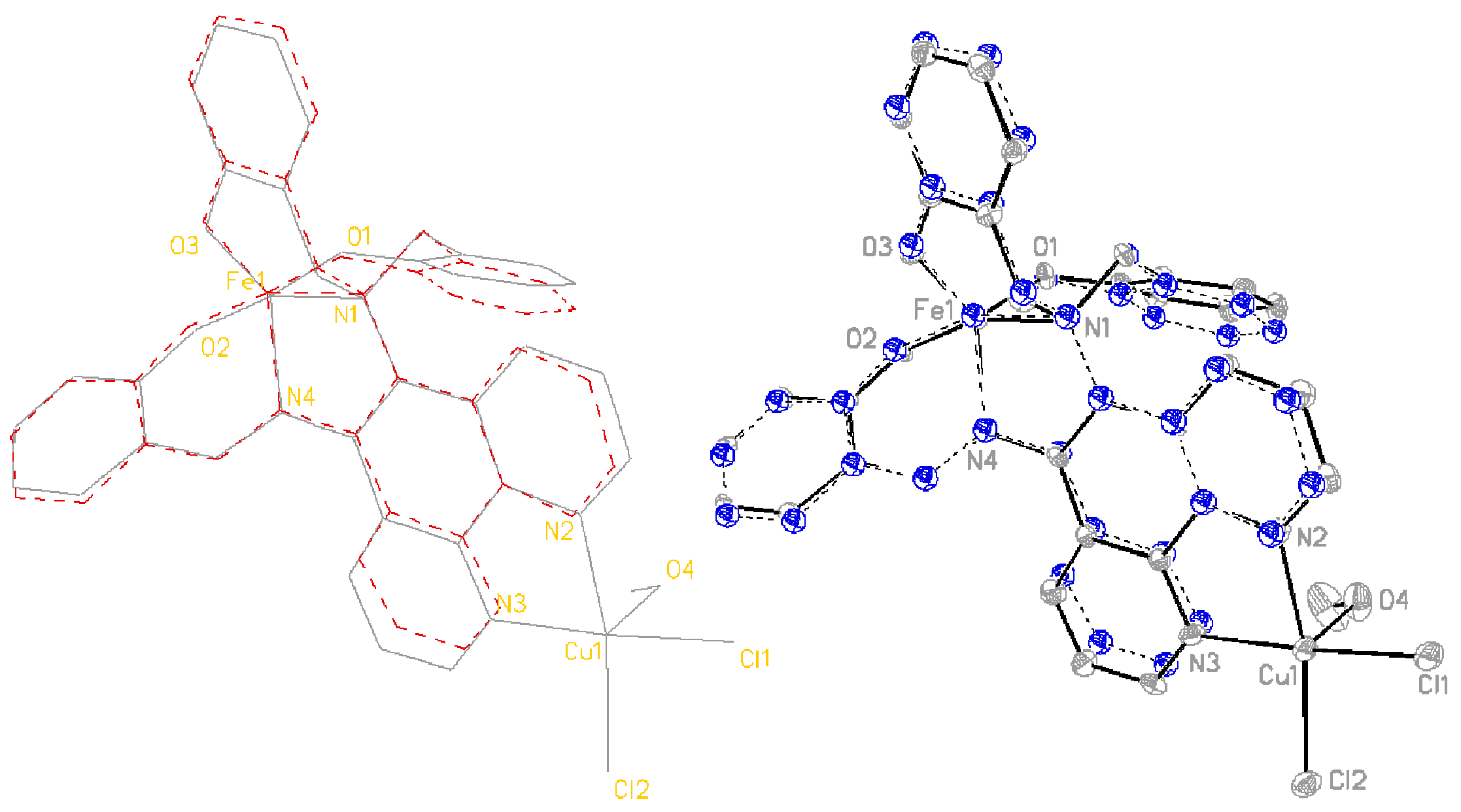




\section{Analysis of the EPR Spectrum for High Spin Fe(III) centers.}

The EPR spectra of high spin Fe(III), $S=5 / 2$, powder samples can be very complex, but become simpler when the zero field interactions have magnitudes greater than the photon energy of the EPR spectrometer. This behavior is demonstrated in Figures 2S, 3S and 4S, where simulations are presented for various relevant situations for an X-band EPR spectrometer. These spectra were calculated assuming using the spin Hamiltonian given in the paper with $g=2.0$ and various values of $D$ and $E$ and $a$ spectrometer frequency of $9.75 \mathrm{GHz}$. The fourth order spin terms were taken to be zero. The $D, E$ values are listed in gauss and the relevant value for the photon energy in gauss is 3500 , so values of D greater than 3500 gauss have zero field interactions greater than the Zeemann energy of the system.

In Figure 2S, we have plotted spectra for the maximum magnitude of $E$, which is one-third of D. Note that for $D$ values below 3500 gauss, the spectra show many features from $B=0$ to 10000 gauss but for higher $D$ values only the peak at $\mathrm{g}=4.3$ is present. At higher gain a second turning point is noted at $\mathrm{g}$ $=8$. This single peak at $g=4.3$ has been observed frequently for biological samples containing high spin $\mathrm{Fe}(\mathrm{III})$.

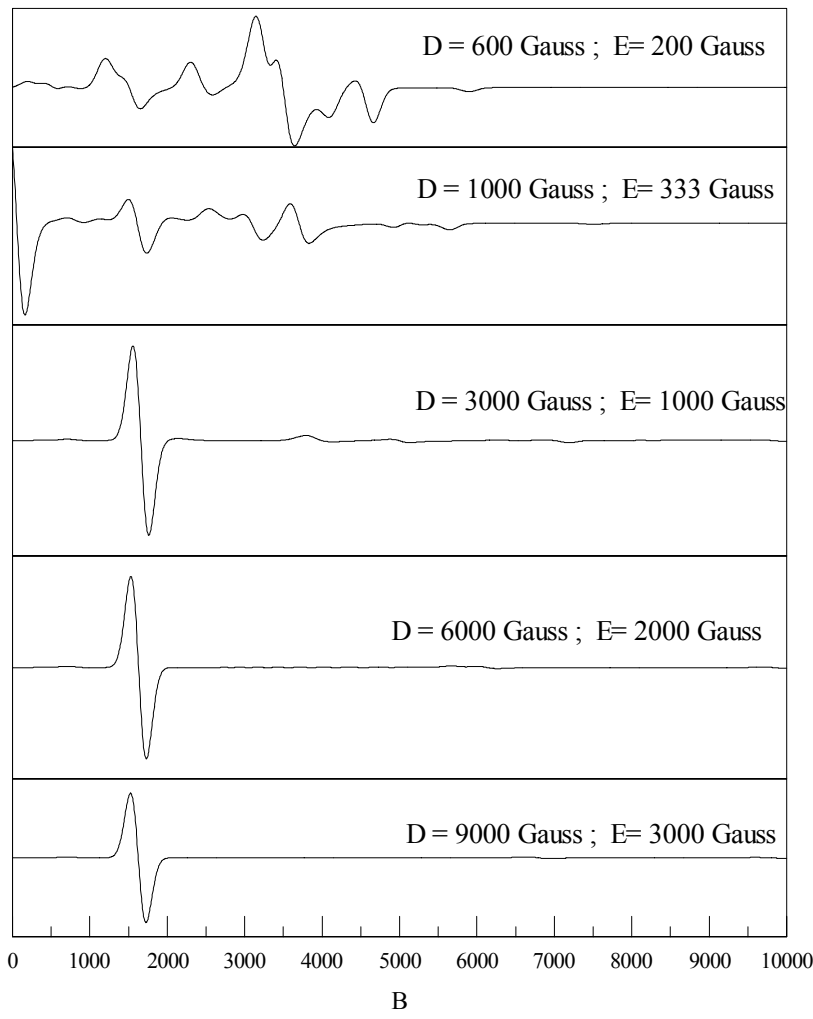

Figure S2. Fe(III) EPR spectra simulated for $E=D / 3$ case. The peak at 1600 gauss $(g \sim 4.3)$ persists as $D$ increases. Notice the major changes when $D$ is less than 3000. Simulation assumes X-band frequency of $9.75 \mathrm{GHz}$ (3500 gauss). 
In Figure 3S, we have plotted spectra for different $D$ values but the symmetry has been taken as axial where $E=0$. In this case the spectrum is dominated by one peak when $D$ is much greater than 3500 gauss but it has a $\mathrm{g}$ value of 6 instead of 4.3. This spectrum is commonly observed in $\mathrm{Fe}$ (III) porphyrins.

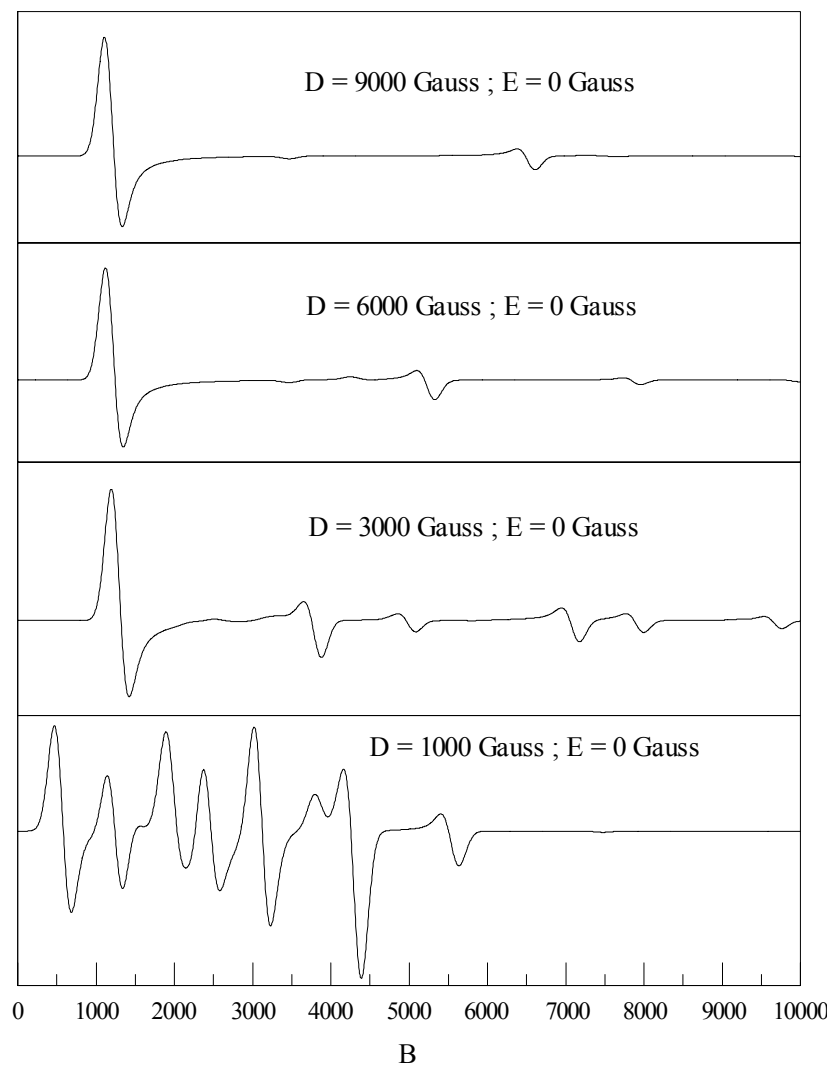

Figure S3. Figure shows how EPR spectrum of $\mathrm{Fe}(\mathrm{III})$ changes with $\mathrm{D}$ when $\mathrm{E}=0$. Note that the peak at 1200 gauss $(g \sim 6)$ remains fixed above $D=$ 3000 gauss. This continues to remain there for much larger D values. It is the only peak detected in Fe(III) porphyrins. 
In Figure 4S, we observe the effect of varying $E$ for a value of $D=6000$ gauss, larger than 3500 gauss. It will be noted that as $E$ is reduced from it's maximum value of $D / 3$ the $g=4.3$ line becomes asymmetric and splits into multiple lines. Also the intensity off the $g$ $=8$ turning point strengthens relative to the main peak at $g=4.3$. This mainly is what we observe in our Fe(III) spectrum and thus leads us to propose that $D$ is much greater than $0.3 \mathrm{~cm}^{-1}$ and that the magnitude of $E$ is slightly less than $D / 3$.

In this system the only way to get good values for the zero field parameters is to study the EPR spectra over a larger range of magnetic fields with spectrometer frequencies much larger than that of an X-band spectrometer.

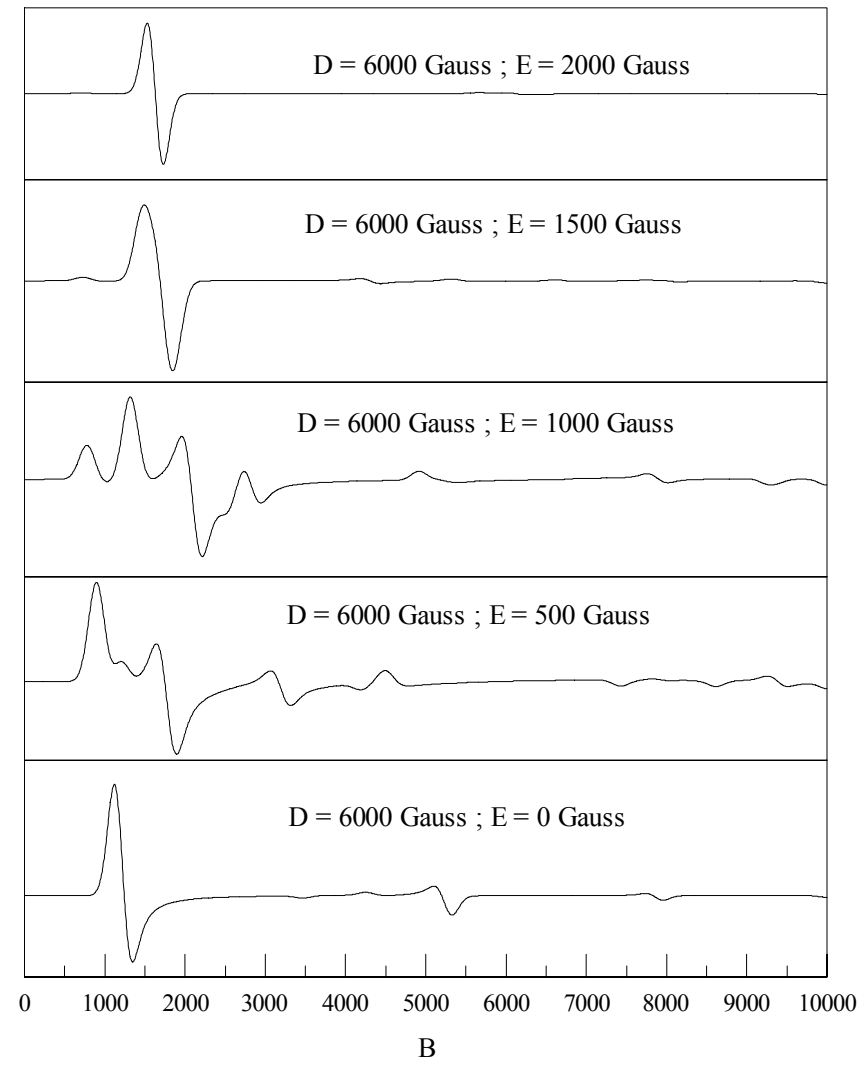

Figure S4. Figure shows simulated Fe(III) spectra for different values of $E$ with $D=6000$ gauss to maximum $E$ value of $D / 3=2000$ gauss. Note that semi peak at 800 gauss becomes more prominent at $E=1500$ gauss . 
Figures S5 and S6: Infrared Spectrum for 3.

From 4000 to $400 \mathrm{~cm}^{-1}$

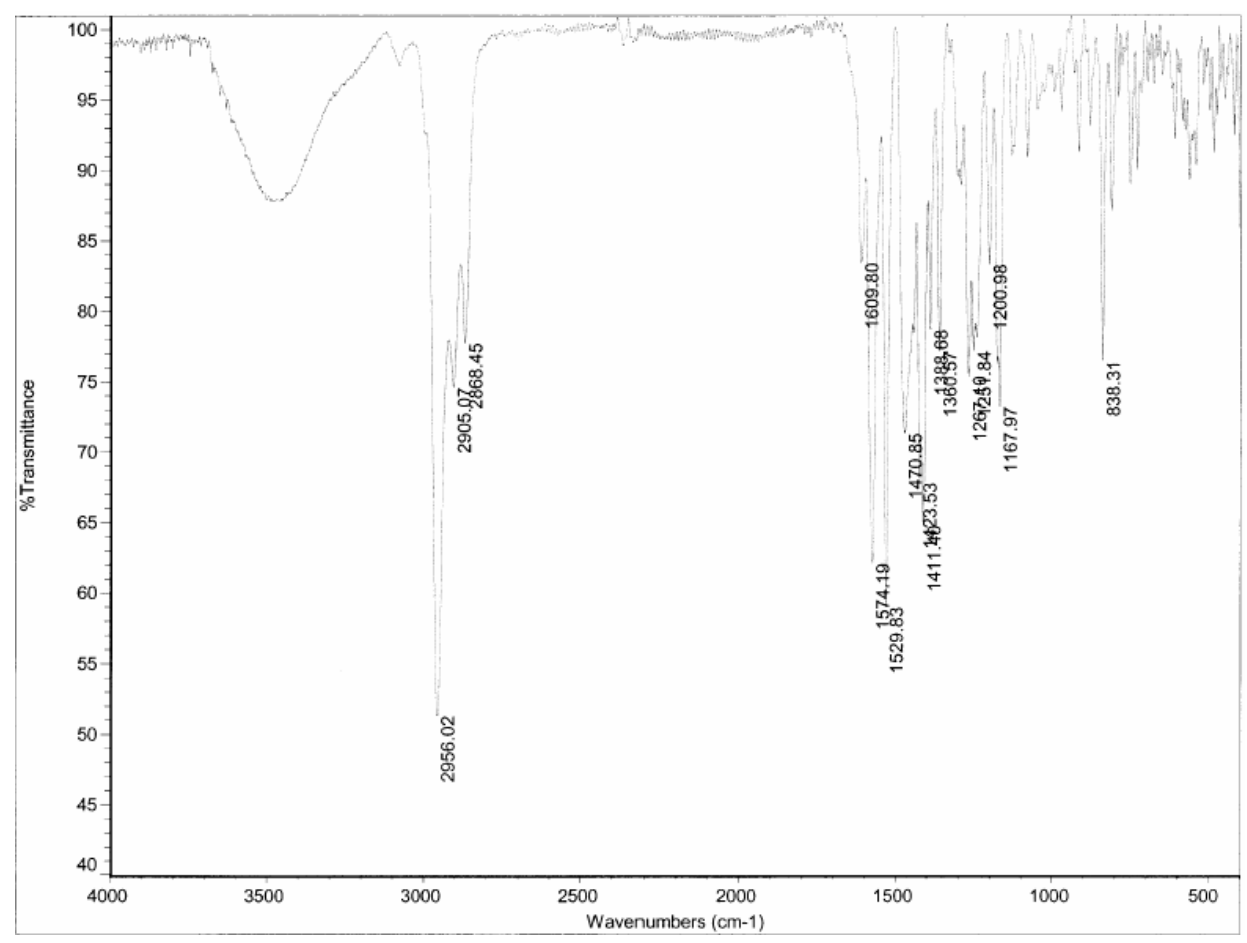

From 1800 to $400 \mathrm{~cm}^{-1}$

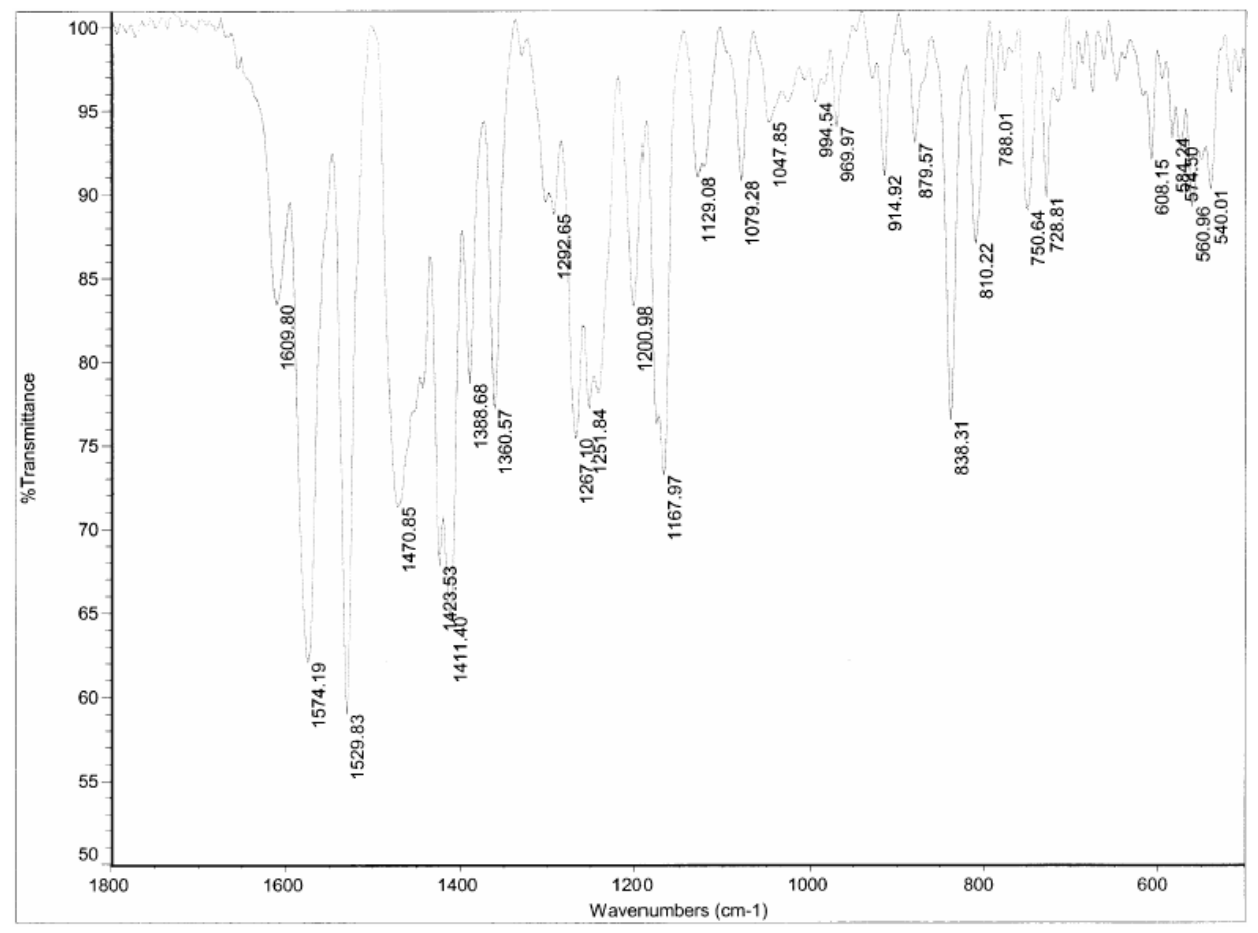

